\title{
Prognostic Factors of Orbital Fractures with Muscle Incarceration
}

\author{
Seung Chan Lee, Seung-Ha Park, Seung-Kyu Han, Eul-Sik Yoon, Eun-Sang Dhong, \\ Sung-Ho Jung, Hi-Jin You, Deok-Woo Kim \\ Department of Plastic and Reconstructive Surgery, Korea University Medical Center, Seoul, Korea
}

Background Among the various signs and symptoms of orbital fractures, certain clinical findings warrant immediate surgical exploration, including gaze restriction, computed tomographic (CT) evidence of entrapment, and prolonged oculocardiac reflex. Despite proper surgical reconstruction, prolonged complications such as diplopia and gaze restriction can occur. This article evaluated the prognostic factors associated with prolonged complications of orbital fractures with muscle incarceration.

Methods The medical records of 37 patients (37 orbits) with an orbital fracture with muscle incarceration from January 2001 to January 2015 were reviewed. The presence of Incarcerated muscle was confirmed via CT, as well as by intraoperative findings. Various factors potentially contributing to complications lasting for over 1 year after the injury were categorized and analyzed, including age, cause of injury, injury-to-operation time, operative time, fracture type, nausea, vomiting and other concomitant symptoms and injuries.

Results All patients who presented with extraocular muscle limitations, positive CT findings, and/or a positive forced duction test underwent surgery. Of the 37 patients, 9 (24\%) exhibited lasting complications, such as diplopia and gaze restriction. The mean follow-up period was 18.4 months (range, 1-108 months), while that of patients who experienced prolonged complications was 30.1 months (range, 13-36 months). Two factors were significantly associated with prolonged complications: injury-to-operation time and nausea/ vomiting. Loss of vision, worsening of motility, and implant complication did not occur.

Conclusions Patients who present with gaze limitations, with or without other signs of a blow-out fracture, require a thorough evaluation and emergent surgery. A better prognosis is expected with a shorter injury-to-operation time and lack of nausea and vomiting at the initial presentation.

Keywords Orbital fractures / Diplopia / Prognosis
Correspondence: Deok-Woo Kim Department of Plastic and Reconstructive Surgery, Korea University College of Medicine, 123 Jeokgeum-ro, Danwon-gu, Ansan 15355, Korea

Tel: $+82-31-412-4817$

Fax: +82-31-475-5074

E-mail: deokwookim@gmail.com
No potential conflict of interest relevant to this article was reported.

Received: 11 May 2017 • Revised: 29 Jul 2017 - Accepted: 5 Aug 2017

pISSN: 2234-6163 • elSSN: 2234-6171 • https://doi.org/10.5999/aps.2017.44.5.407 • Arch Plast Surg 2017;44:407-412

\section{INTRODUCTION}

Orbital fractures were initially described by Lang in 1889 and are known as one of the most common types of facial bone frac- ture [1-3]. Although injuries may involve all 4 walls constituting the orbit, they commonly involve the floor and/or the medial wall [4]. Over the past century, numerous studies have characterized various aspects of these injuries, but opinions still vary

Copyright $\odot 2017$ The Korean Society of Plastic and Reconstructive Surgeons

This is an Open Access article distributed under the terms of the Creative Commons Attribution Non-Commercial License (http://creativecommons.org/

licenses/by-nc/4.0/) which permits unrestricted non-commercial use, distribution, and reproduction in any medium, provided the original work is properly cited.

www.e-aps.org 
regarding the indications and timing of surgery and the length of the postoperative follow-up period [5-7]. Orbital fractures with incarcerated muscle may result in long-lasting complications and should be examined thoroughly.

The surgical indications for orbital fractures remain controversial; in particular, disagreement exists about how and when surgery should take place. In rare cases, emergent surgery should be performed. The clinical findings that warrant immediate surgical exploration include gaze restriction, computed tomographic (CT) evidence of muscle entrapment, and prolonged oculocardiac reflex [8-10].

Despite proper surgical reconstruction, prolonged complications such as diplopia and gaze restriction are possible. The aim of this article was to analyze the risk factors associated with long-lasting complications of orbital fractures, as defined by persisting for over 12 months [11,12]. In this retrospective study, we analyzed the clinical symptoms, signs, and specific characterization of the injuries and the treatment thereof, in order to assess possible risk factors associated with prolonged complications.

\section{METHODS}

\section{Patients}

The records of 37 patients, with 37 orbits, in whom the surgical reconstruction of orbital wall fractures was performed from January 2001 to January 2015 were retrospectively reviewed. All patients underwent a thorough evaluation process including (1) vital signs, (2) a physical examination with inspection of the orbit and periorbital tissues, (3) an assessment of concomitant systemic symptoms, (4) plain film X-rays and CT imaging of at least $3 \mathrm{~mm}$ thickness, (5) an ophthalmologic evaluation as part of the initial physical evaluation, and (6) a forced duction test, if the patient successfully cooperated. Patients who had other coexisting direct injuries involving the optic nerve, lacrimal system, eyelid, globe, or any other intracranial lesions were excluded.

\section{Epidemiology}

The mean age among patients with muscle incarceration was $14.6 \pm 8.0$ years (range, $6-43$ years). There were 28 patients under 15 years old and 9 patients over 15 years old. Of the $37 \mathrm{pa}-$ tients, 36 (97.3\%) were male and 1 (2.7\%) was female. Although the numerous studies on orbital fractures have not shown a uniform distribution of patients or a predominance in the population, there was certainly a male predominance in our patient sample. The most common cause of injury was assault (62.2\%, 23 cases), followed by falls $(27.0 \%, 10$ cases) and sports accidents (10.8\%, 4 cases).

\begin{tabular}{|lc|}
\hline Table 1. Patient demographics \\
\hline Characteristic & No. of patients (\%) \\
\hline Age (yr) & \\
$\quad<15$ & $28(75.7)$ \\
$\geq 15$ & $9(24.3)$ \\
Sex & \\
$\quad$ Male & $36(97.3)$ \\
Female & $1(2.7)$ \\
Cause of injury & \\
Assault & $23(62.2)$ \\
Fall & $10(27.0)$ \\
Sports & $4(10.8)$ \\
Location of fracture & \\
Floor & $23(62.2)$ \\
Medial & $5(13.5)$ \\
Inferomedial & $9(24.3)$ \\
Symptoms (nausea/vomiting) & \\
Absent & $24(62.9)$ \\
Present & $13(35.1)$ \\
Injury-to-operation time (hr) & \\
$\leq 24$ & $20(54.1)$ \\
$>24$ & $17(45.9)$ \\
Operative time (min) & \\
$\leq 60$ & $10(27.0)$ \\
$>60$ & $27(73.0)$ \\
\hline &
\end{tabular}

Orbital fractures without orbital rim involvement were present in 23 cases involving the orbital floor, 5 cases involving the medial wall, and 9 cases involving both. Floor fractures were the most common site of the fracture for both pure and impure fracture types, and incarceration of the extraocular muscles was most commonly found in orbital floor fractures [13] (Table 1).

The mean overall follow-up period was 18.4 months (range, 1-108 months), while that of patients who experienced prolonged complications was 30.1 months (range, 13-36 months). This difference was acceptable due to the fact that patients with postoperative discomfort of any kind are prone to longer followup.

\section{Surgical methods}

When patients were admitted to the clinic and evaluated, CT imaging suggesting incarceration and/or an ophthalmologic evaluation including a positive forced duction test were considered an indication for immediate surgery.

All patients underwent immediate surgery under general anesthesia when clinical signs or radiologic findings led incarceration to be suspected. Nevertheless, several patients inevitably had late surgical timing due to their delayed visit to the clinic. Exploration and release of the incarcerated muscles and surrounding soft tissues were performed. Since multiple surgeons were involved, the surgical approach was chosen based on the operating surgeon's preference and the location of the injury. Trans- 
conjunctival (27 patients, 73.0\%) with or without lateral canthotomy, subcilliary (6 patients, 16.2\%) and transcaruncular (7 patients, $18.9 \%)$ approaches were used. Dissection was performed down to the orbital rim and the fractured lesion was fully exposed. Incarcerated extraocular muscles, as well as herniated soft tissue and orbital fat content, were released and decompressed gently. Bony orbits were reconstructed with absorbable mesh plate (26 orbits), titanium reinforced porous polyethylene mesh (9 orbits), or a porous polyethylene implant ( 2 orbits). The forced duction test was performed before surgery, after plate insertion, and after surgery.

\section{Evaluation of complications}

Diplopia and gaze restriction were assessed at every visit, along with the ophthalmologic evaluation. Diplopia was evaluated by an optometrist with the Goldmann diplopia field test and gaze restriction was evaluated clinically along with a photographic assessment. Ophthalmologic assessments were continued throughout the entire follow-up period unless the patient was free of any clinical complications. A total of 9 patients experienced prolonged complications. We defined "prolonged" as no resolution of the complications (diplopia, gaze restriction) for longer than 12 months postoperatively. Various factors potentially contributing to these poor outcomes were categorized and assessed. These factors were: age, cause of injury, location, injury-to-operation time, operative time, fracture type, and concomitant symptoms. A logistic regression model in SPSS version $12.0 \mathrm{~K}$ for Windows (SPSS Inc., Chicago, IL, USA) was used to analyze the correlations among prolonged complications and the possi-

\begin{tabular}{|c|c|c|c|}
\hline Associated factors & Odds ratio & $\begin{array}{l}\text { 95\% Confidence } \\
\text { interval }\end{array}$ & P-value \\
\hline \multicolumn{4}{|l|}{ Age (yr) } \\
\hline$\geq 15$ & 1.83 & $0.351-9.584$ & 0.473 \\
\hline \multicolumn{4}{|l|}{ Cause of injury } \\
\hline Assault & 0.69 & $0.151-3.192$ & 0.639 \\
\hline Fall & 0.71 & $0.121-4.204$ & 0.710 \\
\hline Sports & 3.71 & $0.441-31.263$ & 0.227 \\
\hline \multicolumn{4}{|l|}{ Location of fracture } \\
\hline Floor & 0.69 & $0.151-3.192$ & 0.639 \\
\hline Medial & 2.38 & $0.330-17.172$ & 0.389 \\
\hline Inferomedial & 0.86 & $0.143-5.130$ & 0.866 \\
\hline Nausea/vomiting & 36.8 & $3.716-364.418$ & $0.002^{\mathrm{a})}$ \\
\hline \multicolumn{4}{|c|}{ Injury-to-operation time (hr) } \\
\hline$>24$ & 6.30 & $1.093-36.301$ & $0.039^{\mathrm{a})}$ \\
\hline \multicolumn{4}{|l|}{ Operative time (min) } \\
\hline$>60$ & 1.40 & $0.238-8.240$ & 0.710 \\
\hline
\end{tabular}

ble risk factors. P-values $<0.05$ were considered to indicate statistical significance. A simple logistic regression model was used to evaluate the significance of predictors along with their odds ratios. Then, factors that were statistically significant were evaluated once more with multiple logistic regression to clarify their correlations.

\section{RESULTS}

In our study, a total of 9 patients (24.3\%) had prolonged complications. Diplopia was most common among these patients, affecting $88.9 \%$ ( 8 out of 9 ) of the patients, 2 of whom showed persistent gaze limitation.

The timing of surgery was categorized as immediate $(\leq 24$ hours after the injury) or late ( $>1$ day after the injury), considering the urgent necessity of intervention in patients with a strong likelihood of muscle incarceration. Twenty patients (54.1\%) underwent immediate surgical exploration and reconstruction, 2 of whom (10.0\%) experienced prolonged complications. The surgical intervention for 17 patients $(45.9 \%)$ took place after the first 24 hours; 7 of these patients (41.2\%) experienced prolonged complications. It is also worth mentioning that both patients who underwent surgical intervention more than 7 days after the injury experienced lasting complications.

The logistic regression model showed (1) injury-to-operative time and (2) nausea/vomiting to be clinically associated with prolonged complications to a significant extent $(\mathrm{P}<0.05)$ Other factors, including age, cause of injury, location of the fracture, and operative time did not show statistical significance (Table 2 ). The 2 factors that showed statistical significance were additionally assessed using multiple logistic regression (Table 3). The odds ratios were also analyzed. The odds ratio of nausea and vomiting for prolonged complications was 67.76 , and that of late surgical timing ( $>24$ hours) was 14.49 in the multiple logistic regression analysis. Although not statistically significant, injuries due to sports activities showed an odds ratio of 3.71 for prolonged complications, and the corresponding odds ratio of medial wall orbital fractures was 2.38. An age of 15 years or over showed an odds ratio of 1.83 , and an operative time of more

Table 3. Two risk factors of prolonged complications (multiple logistic regression model)

\begin{tabular}{|lccc|}
\hline Associated factors & Odds ratio & $\begin{array}{c}\text { 95\% Confidence } \\
\text { interval }\end{array}$ & P-value \\
\hline $\begin{array}{l}\text { Nausea/vomiting } \\
\text { Injury-to-operation time (hr) } \\
>24\end{array}$ & 67.76 & $4.129-1111.766$ & $0.003^{\text {a) }}$ \\
\hline a)Statistically significant. & 14.49 & $1.094-191.925$ & $0.043^{\text {a) }}$ \\
\hline
\end{tabular}


Fig. 1. Initial photograph and CT finding of 14-year-old male patient (Case)

$(A, B)$ Preoperative photo of extraocular muscle movement shows significant vertical gaze restriction. (C) Coronal computed tomography shows signs of soft tissue herniation and suspected incarceration of the inferior rectus muscle. CT, computed tomography.
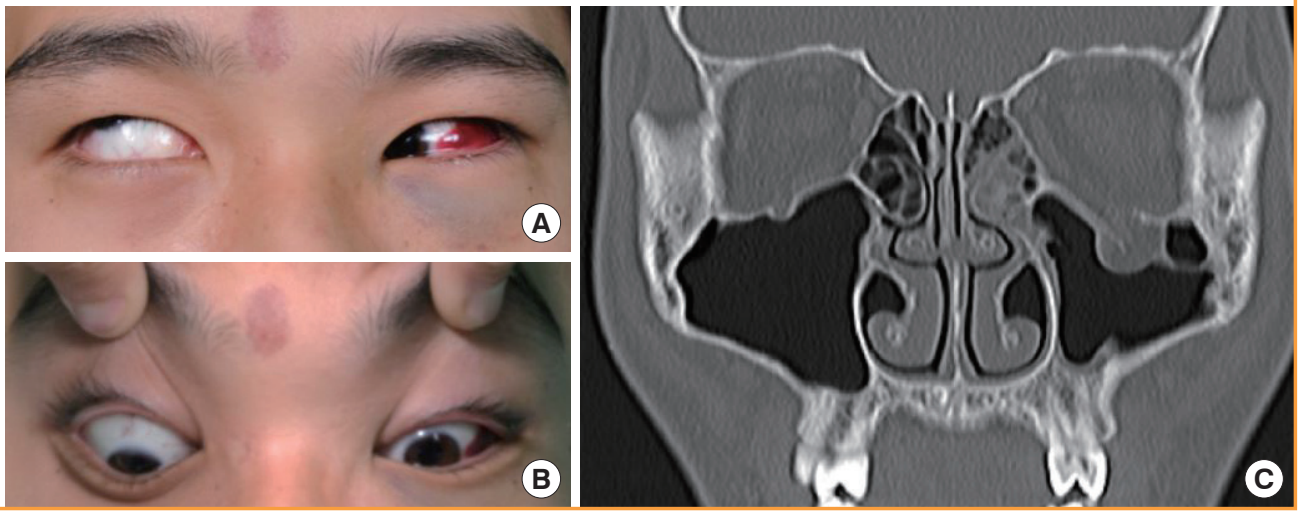

Fig. 2. Photograph and CT finding of 1 year after surgery, same patient shown in Figure 1

(A, B) One-year postoperative photo of extraocular muscle movement shows unresolved restrictions. (C) Coronal computed tomography scans show nonsignificant findings. CT, computed tomography.
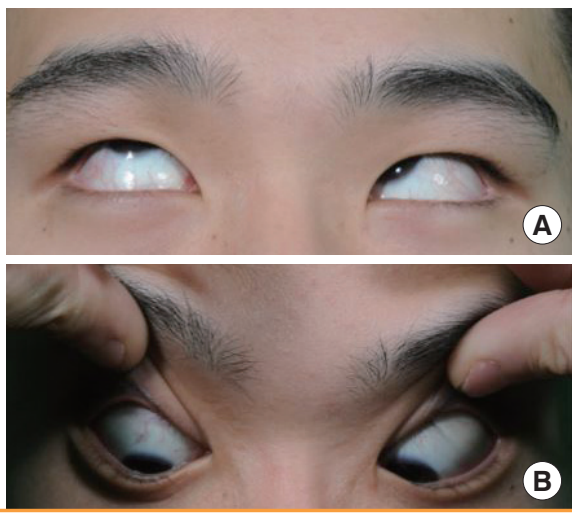

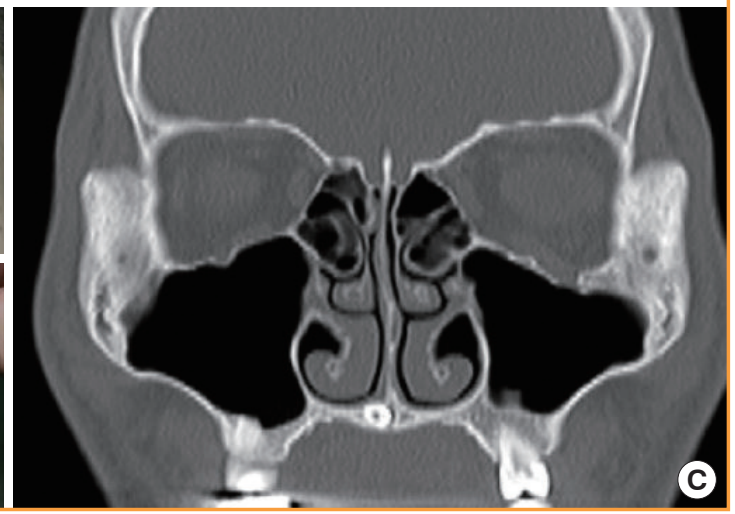

than 60 minutes showed an odds ratio of 1.42 .

The preoperative and postoperative findings of a case demonstrating clinical signs upon initial admission to the clinic and on coronal CT are discussed below.

\section{Case}

A 14-year-old male presented with left ocular pain 10 hours after a fall injury. Although the patient's vital signs were normal, he presented with severe nausea and vomiting (4 episodes). The patient also had subconjunctival hemorrhage, partial sensory loss in the maxillary area, and mild swelling. The patient had no other medical history. Coronal CT (3-mm thickness) revealed signs of a trapdoor fracture of the orbital floor. A physical examination showed vertical gaze restriction along with a positive forced duction test. The patient underwent surgery under general anesthesia 47 hours after the injury via a transconjunctival approach with lateral canthotomy, using titanium-reinforced porous polyethylene mesh. Hypoesthesia of the maxillary region resolved 3 months postoperatively. However, despite proper surgical intervention, he did not show improvement until 27 months after surgery. Based on 4 series of facial bone CT followup, bony reduction with positioning of the titanium-reinforced polyethylene mesh was successful. However, swelling and a poorly defined left inferior rectus muscle remained unchanged (Figs. 1, 2).

\section{DISCUSSION}

Although our retrospective study did not place a restriction on age at the time of the evaluation, the mean age was 14.6 years (range, 6-43 years). This is in accordance with recent studies showing an increased potential for trapdoor fractures in the pediatric age group $[14,15]$. The location of the fracture also had a similar distribution in the pediatric age group as in other age groups, with the orbital floor being the most commonly involved site [7]. In our study, diplopia was the foremost prolonged complication, followed by gaze restriction. This result is supported by the finding that persistent diplopia is more common in children as a result of their unique fracture patterns. Enophthalmos was present in a single patient, but that patient was not considered to have an orbital fracture complication, as enophthalmos is associated with orbital volume expansion, which does not usually occur in orbital fractures with incarceration.

Several studies have demonstrated a close correlation between 
nausea and vomiting after injury and trapdoor fractures $[8,14$, 16]. It is believed that this vagal response is secondary to trapped parasympathetic nerve fibers that travel with the entrapped muscle, sending efferent signals to the stomach and heart. When bradycardia accompanies such symptoms, the oculocardiac reflex is strongly implicated, as described by Sires et al. [14], which can result in rare, but potentially fatal, cardiac arrhythmias. Cohen and Garrett [16] evaluated nausea/vomiting as a sign of entrapment, and those symptoms had a $83.3 \%$ positive predictive value in their study. In our study, such non-ocular symptoms were poor prognostic factors for incarcerated blowout fractures. Not only did those symptoms correlate with trapdoor fractures, as prior articles described, but they strongly suggested the possibility of prolonged diplopia and extraocular muscle limitations. According to our study, having nausea and vomiting at presentation is an extremely strong indication for immediate surgery, as its odds ratio for prolonged complications was greater than 60 . Therefore, the importance of an initial evaluation of nausea, vomiting, and other vagal signs such as bradycardia should be underscored, in addition to the periocular inspection.

Although there were no statistically significant results for radiologic findings, the role of CT studies is worth discussing. CT scans have the highest degree of confidence for diagnosing orbital fractures. However, it is important to recognize the possibility of false negatives, especially among pediatric patients $[9,15]$. Our study found that 13 out of 37 patients $(35.1 \%)$ had nonsignificant radiologic findings, without a clear visualization of the herniated muscles. The CT findings were inconclusive in a significant number of patients. For pediatric patients in particular, greenstick fractures can leave only minimal fracture lines, or even none, on coronal CT because the fracture line can be aligned with the coronal section or can simply be missed in images with thickness of a few millimeters. Moreover, pediatric patients can be very irritable due to pain, complicating attempts to perform an adequate clinical evaluation.

White-eyed orbital fractures were first described by Jordan et al. [15], who described blow-out fractures without soft tissue trauma (e.g., edema or ecchymosis), diplopia with vertical gaze restriction, lack of enophthalmos, and most importantly, an absence of radiologic signs of muscle entrapment. In another report, Wachler and Holds [17] introduced the "missing muscle syndrome," in which CT examinations failed to capture muscle entrapment requiring emergent surgery. Therefore, a multidisciplinary approach should be used when muscle entrapment is suspected, especially in pediatric patients.

There are differing opinions on the timing of surgery. Dutton [18] suggested relatively early surgical interventions, within the first 2 weeks in cases of symptomatic diplopia with a positive forced duction test, radiologic findings of entrapment, early enophthalmos of $3 \mathrm{~mm}$ or more, and large orbital wall defects likely to result in late enophthalmos. Although such early surgical interventions in cases of suspected incarceration are becoming increasingly accepted in comparison with the traditional 'wait and see' option, there are inconsistent views on the best timing for surgical interventions [19]. To our knowledge, it is safe to emphasize the importance of immediate surgical intervention in the first 24 hours after injury in order to obtain optimal results and faster recovery. Immediate surgery can avoid possible muscle ischemia followed by fibrosis and contracture of the surrounding soft tissue content, which consequently leads to decreased muscle motility [20]. For patients with orbital fractures with suspected muscle incarceration, delayed surgery can lead to prolonged complications, and for patients who inevitably receive late surgical care due to delayed admission to the clinic, prompt warnings about potential complications are crucial.

Orbital fractures are one of the most common types of facial bone fractures. Among the various symptoms and signs, periocular injuries with limited extraocular muscle movement deserve closer attention. False-negative findings can cause devastating results. Nausea and vomiting at presentation are closely associated with orbital fractures that are significant enough to consider other diagnostic tools such as magnetic resonance imaging or the forced duction test under general anesthesia, as many pediatric patients cannot comply with such invasive physical examinations after an injury. Prompt evaluation when entrapment is suspected and immediate surgery within the first 24 hours are crucial factors in the diagnosis and treatment of orbital fractures.

Our study has some limitations due to its relatively small sample size. In particular, there were not many patients who had a surgical intervention 7 days or more after the injury. Moreover, the relatively short follow-up period and the presence of less clinical data, such as postoperative CT scans, among patients without any complications may need further exploration. Thus, larger long-term prospective studies should be conducted to further characterize these specific injuries.

A better prognosis is expected with a shorter injury-to-operation time, preferably within the first 24 hours after the injury, and a lack of nausea and vomiting at the initial presentation. Radiologic findings are important for the diagnosis but should not be relied upon exclusively.

\section{PATIENT CONSENT}

The patient provided written informed consent for the publication and the use of their images. 


\section{REFERENCES}

1. Lang W. Injuries and diseases of the orbit: traumatic enophthalmos with retention of perfect acuity of vision. By William Lang, 1889. Adv Ophthalmic Plast Reconstr Surg 1987;6:3-6.

2. Hosal BM, Beatty RL. Diplopia and enophthalmos after surgical repair of blowout fracture. Orbit 2002;21:27-33.

3. Pfeiffer RL. Traumatic enophthalmos. Trans Am Ophthalmol Soc 1943;41:293-306.

4. Ellis E 3rd, el-Attar A, Moos KF. An analysis of 2,067 cases of zygomatico-orbital fracture. J Oral Maxillofac Surg 1985; 43:417-28.

5. Cole P, Boyd V, Banerji S, et al. Comprehensive management of orbital fractures. Plast Reconstr Surg 2007;120:57S63S.

6. Burnstine MA. Clinical recommendations for repair of isolated orbital floor fractures: an evidence-based analysis. Ophthalmology 2002;109:1207-10.

7. Cruz AA, Eichenberger GC. Epidemiology and management of orbital fractures. Curr Opin Ophthalmol 2004;15: 416-21.

8. Baek SH, Lee EY. Clinical analysis of internal orbital fractures in children. Korean J Ophthalmol 2003;17:44-9.

9. Grant JH 3rd, Patrinely JR, Weiss AH, et al. Trapdoor fracture of the orbit in a pediatric population. Plast Reconstr Surg 2002;109:482-9.

10. Kang MS, Jeong EC. White-eyed medial orbital wall fracture with oculocardiac symptoms. Arch Plast Surg 2015;42:2479.
11. Hawes MJ, Dortzbach RK. Surgery on orbital floor fractures. Influence of time of repair and fracture size. Ophthalmology 1983;90:1066-70.

12. Biesman BS, Hornblass A, Lisman R, et al. Diplopia after surgical repair of orbital floor fractures. Ophthal Plast Reconstr Surg 1996;12:9-16.

13. Bansagi ZC, Meyer DR. Internal orbital fractures in the pediatric age group: characterization and management. Ophthalmology 2000;107:829-36.

14. Sires BS, Stanley RB Jr, Levine LM. Oculocardiac reflex caused by orbital floor trapdoor fracture: an indication for urgent repair. Arch Ophthalmol 1998;116:955-6.

15. Jordan DR, Allen LH, White J, et al. Intervention within days for some orbital floor fractures: the white-eyed blowout. Ophthal Plast Reconstr Surg 1998;14:379-90.

16. Cohen SM, Garrett CG. Pediatric orbital floor fractures: nausea/vomiting as signs of entrapment. Otolaryngol Head Neck Surg 2003; 129:43-7.

17. Wachler BS, Holds JB. The missing muscle syndrome in blowout fractures: an indication for urgent surgery. Ophthal Plast Reconstr Surg 1998; 14:17-8.

18. Dutton JJ. Management of blow-out fractures of the orbital floor. Surv Ophthalmol 1991;35:279-80.

19. Kim JW, Bae TH, Kim WS, et al. Early reconstruction of orbital roof fractures: clinical features and treatment outcomes. Arch Plast Surg 2012;39:31-5.

20. Egbert JE, May K, Kersten RC, et al. Pediatric orbital floor fracture: direct extraocular muscle involvement. Ophthalmology 2000;107:1875-9. 